\title{
V.V. Chistyakov
}

National Research University Higher School of Economics, Nizhny Novgorod, Russia

K.O. Chumakova

National Research University Higher School of Economics,

Nizhny Novgorod, Russia

\section{Restoring Indifference Classes via Ordinal Numbers under the Discrete Leximin and Leximax Preference Orderings}

Abstract. The leximin (leximax) preference ordering compares two $n$-dimensional real vectors as follows: the coordinates of these vectors are first ordered in ascending (descending) order and then the resulting two vectors are compared lexicographically. It is well known that the leximin (leximax) preference ordering on $\mathbb{R}^{n}$ is not representable (by a utility function). In this paper, given two integers $n \geq 1$ and $m \geq 2$, we consider the set $X$ of all $n$-dimensional vectors with integer coordinates assuming values between 1 and $m$. Equipping $X$ with the leximin (leximax) preference ordering induced from $\mathbb{R}^{n}$, called the threshold (dual threshold) rule, every vector from $X$ (and its indifference class) is canonically assigned a unique ordinal number in such a way that a vector from $X$ is considered more leximin- (leximax-) preferable if it lies in an indifference class with greater ordinal number. We present a rigorous recursive algorithm for the evaluation of multiplicities of the coordinates in a vector from $X$ via the ordinal number of the indifference class with respect to the ordering, to which this vector belongs. The novelty of our algorithm is twofold: first, it exhibits new properties of the classical binomial coefficients in their interplay with the leximin (leximax) preference ordering and, second, it relies on four integer parameters, each one being obtained by a different cyclic procedure. The joint work of these procedures is based on our main theorem concerning some subtle properties of the enumerating preference function, which represents the leximin (leximax) preference ordering on $X$.

Keywords: weak order, indifference class, lexicographic preference, leximin, leximax, ordinal number, enumerating preference function.

JEL Classification: C020, C810, D790, E190.

DOI: $10.31737 / 2221-2264-2018-39-3-1$

\section{Introduction}

The paper addresses the following problem. Suppose $X$ is the set of all $n$-dimensional vectors $x=\left(x_{1}, \ldots, x_{n}\right)$ with integer coordinates $1 \leq x_{i} \leq m$ for some integer $m \geq 2$. We equip $X$ with the leximin (leximax) preference ordering induced from $\mathbb{R}^{n}$. Recall that, under the leximin (leximax), the coordinates of two vectors are first ordered in ascending (descending) order and then the resulting two vectors are compared lexicographically. The leximin (leximax) preference ordering partitions $X$ into indifference (= equivalence) classes, each of which being uniquely assigned an ordinal number with respect to the ordering in such a way that a vector from $X$ is considered more leximin- (leximax-) preferable if it lies in an indifference class with greater ordinal number. The problem is to restore the whole indifference class by knowing its ordinal number.

\footnotetext{
${ }^{1}$ The authors express their sincere gratitude to Professor F.T. Aleskerov (NRU HSE, Moscow) for several stimulating discussions, concerning this paper, and encouragement.
} 
The lexicographic, leximin and leximax preference orderings in different contexts have been studied extensively in the literature, mainly in the axiomatic approach (Bridges, Mehta, 1995; Chistyakov, 2014; d'Aspremont, Gevers, 2002; Fishburn, 1970, 1975, 1999; Moulin, 1988; Sen, 1970; Vilkas, 1986). In general, no utility function exists for these preference orderings.

In this paper, we shall be interested in the leximin (leximax) preference ordering, restricted to the above set $X$ of $n$-dimensional vectors. In this case, the interval of natural numbers $[1, n]=\{1, \ldots, n\}$ can be interpreted as the set of agents, number $x_{i}$ (between 1 and $m$ ) - as the $i$-th agent's grade, $i \in[1, n]$, and vector $x=\left(x_{1}, \ldots, x_{n}\right) \in X-$ as an alternative estimated by the agents from $[1, n]$. The preference ordering on $X$, corresponding to the leximin (leximax), which is originated from (Aleskerov, Yakuba, 2007; Aleskerov, Yakuba, Yuzbashev, 2007) for $m=3$, is called the threshold rule (dual threshold rule). The complete axiomatic characterization of the rule(s) for arbitrary integer $m \geq 3$ was given in (Aleskerov et al., 2010a, 2010b); different perspectives of the rule(s) were considered in (Podinovskii, 1975).

The axioms used in the characterization of the threshold rule are Pairwise Compensation, Pareto Domination, Noncompensatory Threshold and Contraction. In order to get a better feeling of the threshold rule, we present a small citation from (Aleskerov et al., 2010b, p. 628-629).

"The Pairwise Compensation axiom means that if all agents, but two, evaluate two alternatives equally, and the two agents put 'mutually inverse' grades, then the two alternatives have the same rank in the social decision (which may be interpreted as 'anonymity of grades').

The Pareto Domination axiom states that if the grades of all agents for one alternative are not less than for the second alternative and the grade of at least one agent for the first alternative is strictly greater than that of the second one, then in the social ranking the first alternative has a higher rank than the second alternative.

The Noncompensatory Threshold axiom reveals the main idea of the threshold aggregation: if at least one agent evaluates an alternative as 'bad', then, no matter how many 'good' grades it admits, in the social ranking this alternative is ranked lower than any alternative evaluated as 'average' by all agents.

In this context, the Contraction means that if for two alternatives the evaluations of some agent are equal, then the agent may be 'excluded' from the consideration when the social ranking is constructed, and the social decision is achieved by the remaining agents' evaluations."

The real-life applications of the threshold rule were presented in (Aleskerov et al., 2014) for the evaluation of the bank branch performance and (Goncharov, Chistyakov, 2012) for noncompensatory ranking of students of the National Research University Higher School of Economics (to mention a few).

In contrast to the general case of the leximin (leximax) on $\mathbb{R}^{n}$, for the threshold preference ordering of $X$, a surjective utility function 
of the form $\Phi: X \rightarrow[1, s]=\{1, \ldots, s\}$ exists for some integer $s$. Interestingly, the explicit formula for $\Phi$ can be given combinatorially in terms of binomial coefficients (see (Aleskerov, Chistyakov, 2013b; Chistyakov, 2009; Chistyakov, Kalyagin, 2008) and Theorem 1 in Section 2). Since $\Phi$ represents the leximin (leximax) on $X$, the collection $\left\{X_{\ell}: \ell \in[1, s]\right\}$ of counterimages $X_{\ell} \equiv \Phi^{-1}(\ell)=\{x \in X: \Phi(x)=\ell\}$ is exactly the family of all indifference classes with respect to the leximin (leximax) preference ordering. In other words, the problem above can be reformulated in terms of 'inversing' the discrete function $\Phi$ as follows: given $\ell \in[1, s]$, restore the class $X_{\ell}=\Phi^{-1}(\ell)$. So, knowing the ordinal number of (the indifference class of) the alternative $x \in X$ it will be possible to obtain the whole collection of agents' grades $x_{1}, \ldots, x_{n}$. To the best of our knowledge, it is the first time in the literature that the aggregation procedure (which is the threshold rule based on the leximin on $X$ ) permits to return back the rather 'big' input data $x_{1}, \ldots, x_{n}$ by means of the relatively 'small' aggregate ordinal number of $x$.

We do not aim at the applications in this paper, concentrating on the mathematical problem of inversing the 'discrete' function $\Phi$. The reallife applications are postponed until subsequent publications.

We solve the problem of restoring the indifference classes by presenting a rigorous recursive algorithm. Its novelty is twofold. First, it exhibits new properties of the classical binomial coefficients in their interplay with the leximin (leximax) preference ordering. Second, the algorithm involves four integer parameters $n, m, L$, and $\ell$ (indexed by $j \in[1, m]$ ), each one being obtained by a different procedure: $n$ is obtained through the decomposition of the natural 'interval' $[1, s]$ into a disjoint union of smaller intervals, $m$ - by subtracting $1, L-$ by putting $n$ and $m$ into a binomial coefficient, and the 'new' value of $\ell$ - by subtracting $L$ from the 'previous' value of $\ell$. This 'cyclic procedure' is made possible to work successfully by our main result, Theorem 3 , concerning some subtle properties of the function $\Phi$.

The paper is organized as follows. In Section 2, we review certain definitions and auxiliary facts and recall explicit formulas for the enumerating preference functions representing the leximin/leximax preference ordering on $X$ (Theorems 1 and 2). Our main result, Theorem 3 in Section 3 , is devoted to the evaluation of multiplicities of coordinates in a given vector from $X$. The algorithm for restoring indifference classes in the case of the leximin preference ordering on $X$, based on Theorem 3, is presented in Section 4 and illustrated by an example. Finally, in Section 5, the restoring of indifference classes in the case of the leximax is reduced to the case of the leximin preference ordering and the corresponding algorithm.

\section{Preliminaries}

We begin by reviewing certain definitions and facts needed for our results. 
Throughout the paper $n$ is a positive integer and $\mathbb{R}^{n}$ is the set of all $n$-dimensional vectors $x=\left(x_{1}, \ldots, x_{n}\right)$ with real coordinates $x_{i} \in \mathbb{R}$. Given two nonnegative integers $k$ and $l$ with $k \leq l$, the set $\{k, k+1, \ldots, l\}$ is denoted by $[k, l]$ and said to be the (natural) interval with endpoints $k$ and $l$ (if $k>l$, we set $[k, l]=\varnothing)$. Of main interest below is the set $X=[1, m]^{n} \subset \mathbb{R}^{n}$, with integer $m \geq 2$, of all $n$-dimensional vectors $x=\left(x_{1}, \ldots, x_{n}\right)$ with coordinates $x_{i} \in[1, m]$. The elements of $[1, m]$ may be interpreted as ordered grades $1<2<\ldots<m-1<m$, and the elements of $[1, n]-$ as agents (criteria, parameters, entities, or properties). The number of elements in $[1, m]^{n}$ is equal to $\left|[1, m]^{n}\right|=m^{n}$.

A vector $y \in \mathbb{R}^{n}$ is said to be lexicographically preferred to $x \in \mathbb{R}^{n}$ (in symbols, $\left.x \angle_{n} y\right)$ provided $x_{1}<y_{1}$, or there is $k \in[2, n]$ such that $x_{i}=y_{i}$ for all $i \in[1, k-1]$ and $x_{k}<y_{k}$. It is well known (Fishburn 1970, 1975) that the lexicographic preference $\angle_{n}$ on $\mathbb{R}^{n}$ is transitive (i.e., $x \angle_{n} y$ and $y \angle_{n} z$ imply $x \angle_{n} z$ ), the negation of $\angle_{n}$ is of the form: $\neg\left(x \angle_{n} y\right)$ iff (=if and only if) $y \angle_{n} x$ or $y=x$, and $\angle_{n}$ is trichotomous (i.e., either $x \angle_{n} y$, or $y \angle_{n} x$, or $x=y$ ). Thus, $\angle_{n}$ is a linear order on $\mathbb{R}^{n}$.

Putting the coordinates of $x=\left(x_{1}, \ldots, x_{n}\right) \in \mathbb{R}^{n}$ in ascending (respectively, descending) order, we denote the resulting vector by $x^{*}=\left(x_{1}^{*}, \ldots, x_{n}^{*}\right)$ with $x_{1}^{*} \leq \ldots \leq x_{n}^{*}$ (resp., by $x_{*}=\left(x_{*_{1}}, \ldots, x_{*_{n}}\right)$ with $\left.x_{*_{1}} \geq \ldots \geq x_{*_{n}}\right)$ and call it the nondecreasing (respectively, nonincreasing) representative of $x$. If $X$ is a nonempty subset of $\mathbb{R}^{n}$, we set $X^{*}=\left\{x^{*}: x \in X\right\}$ and $X_{*}=\left\{x_{*}: x \in X\right\}$.

A vector $y \in \mathbb{R}^{n}$ is said to be preferred to $x \in \mathbb{R}^{n}$ in the sense of the leximin (resp., leximax) provided $x^{*} \angle_{n} y^{*}$, also written as $x \angle_{n}^{*} y$ (resp., $x_{*} \angle_{n} y_{*}$, also written as $x \angle_{*_{n}} y$ ). It is well known (Bridges, Mehta, 1995; Fishburn, 1970; Sen, 1970; Vilkas, 1986) that the lexicographic ordering $\angle_{n}$, as well as the leximin $\angle_{n}^{*}$ and leximax $\angle_{*}$ preference orderings, are not representable on $\mathbb{R}^{n}$, i.e., there is no (utility) function $\varphi: \mathbb{R}^{n} \rightarrow \mathbb{R}$ such that, given $x, y \in \mathbb{R}^{n}, x \angle_{n} y$ iff $\varphi(x)<\varphi(y)$. It is to be noted that $\angle_{n}^{*}$ and $\angle_{*_{n}}$ are not linear orders on $\mathbb{R}^{n}$ : if we set $(x, y) \in P$ iff $y \angle_{n}^{*} x$ (or $(x, y) \in P$ iff $y \angle_{*_{n}} x$ ), then $P$ is a weak order on $\mathbb{R}^{n}$ in the following sense (Aleskerov, Bouyssou, Monjardet, 2007).

A binary relation $P$ on a set $X$ is said to be a weak order on $X$ if it has the following three properties, for all $x, y, z \in X$ :

(i) $(x, y) \in P$ and $(y, z) \in P$ imply $(x, z) \in P$ (transitivity);

(ii) $(x, x) \notin P$ (irreflexivity);

(iii) $(x, y) \notin P$ and $(y, z) \notin P$ imply $(x, z) \notin P$ (negative transitivity).

The inclusion $(x, y) \in P$ is conventionally interpreted as ' $x$ is preferred to $y^{\prime}$ (with respect to $P$ ). The indifference relation $I_{P}$ on $X$, induced 
by $P$, is defined as usual by: given $x, y \in X,(x, y) \in I_{P}$ iff $(x, y) \notin P$ and $(y, x) \notin P$. Clearly, $I_{P}$ is an equivalence relation on $X$.

A weak order $P(\varphi)$ on a set $X$, generated by a nonconstant function $\varphi: X \rightarrow \mathbb{R}$, is defined as follows: given $x, y \in X$, set $(x, y) \in P(\varphi)$ iff $\varphi(x)>\varphi(y)$. Clearly, $(x, y) \in I_{P(\varphi)}$ iff $\varphi(x)=\varphi(y)$. A binary relation $P$ on $X$ is said to be representable (by $\varphi$ ) if $P=P(\varphi)$, in which case $\varphi$ is called a preference (or util ity) function for $P$ (note that preference functions for $P$ are determined nonuniquely in general).

From now on, $X \equiv X_{m, n}=[1, m]^{n}$ with integers $n \geq 1$ and $m \geq 2$.

The monotone representatives of $x=\left(x_{1}, \ldots, x_{n}\right) \in[1, m]^{n}$ are of the form

$$
\begin{aligned}
& x^{*}=\left(1^{v_{1}(x)}, 2^{v_{2}(x)}, \ldots,(m-1)^{v_{m-1}(x)}, m^{v_{m}(x)}\right), \\
& x_{*}=\left(m^{v_{m}(x)},(m-1)^{v_{m-1}(x)}, \ldots, 2^{v_{2}(x)}, 1^{v_{1}(x)}\right),
\end{aligned}
$$

where $v_{j}(x)=\left|\left\{i \in[1, n]: x_{i}=j\right\}\right|$ is the multiplicity of grade $j \in[1, m]$ in $x$, and $j^{v_{j}(x)}$ denotes $j, j, \ldots, j$ repeated $v_{j}(x)$ times (if $v_{j}(x)=0$, the term $j^{v_{j}(x)}$ is omitted in the notation of $x^{*}$ and $\left.x_{*}\right)$. Note at once that $v_{j}\left(x^{*}\right)=v_{j}\left(x_{*}\right)=v_{j}(x)$ for all $j \in[1, m]$ and

$$
v_{1}(x)+\ldots+v_{m}(x)=n \text { for all } x \in[1, m]^{n} .
$$

It was shown in (Aleskerov, Chistyakov, Kalyagin, 2010b) that if $X=[1, m]^{n}$, then

$$
\left|X^{*}\right|=\left|X_{*}\right|=C_{n+m-1}^{m-1}=C_{n+m-1}^{n}=\frac{(n+m-1) !}{n !(m-1) !}
$$

where $C_{n}^{k} \equiv\left(\begin{array}{l}n \\ k\end{array}\right)=n ! /[k !(n-k) !]$ is the usual binomial coefficient if $k \in[0, n]$, and $0 !=1$.

The leximin $\angle_{n}^{*}$ and leximax $\angle_{*_{n}}$ preference orderings on $X=[1, m]^{n}$ can be given alternative characterizations (more suitable for our purposes) as follows. Given $x \in X$, define two vectors $v(x)$ and $\bar{v}(x)$ from $[0, n]^{m-1}$ by $v(x)=\left(v_{1}(x), v_{2}(x), \ldots, v_{m-1}(x)\right)$ and $\bar{v}(x)=\left(v_{m}(x), v_{m-1}(x), \ldots, v_{2}(x)\right)$. The threshold preference order $P_{m-1}$ (resp., dual threshold preference order $\bar{P}_{m-1}$ ) on $X=[1, m]^{n}$, which is a weak order on $X$, is defined, for $x, y \in X$, by (Aleskerov, Yakuba, Yuzbashev, 2007; Aleskerov, Chistyakov, Kalyagin, 2010b)

$$
\left.(x, y) \in P_{m-1} \text { iff } v(x) \measuredangle_{m-1} v(y) \quad \text { (resp., }(x, y) \in \bar{P}_{m-1} \text { iff } \bar{v}(y) \measuredangle_{m-1} \bar{v}(x)\right) .
$$

It was shown by (Aleskerov, Chistyakov, 2013b) that

$$
y \angle_{n}^{*} x \text { iff }(x, y) \in P_{m-1} \text {, and } y \angle_{* n} x \text { iff }(x, y) \in \bar{P}_{m-1} .
$$

In what follows, we treat $\angle_{n}^{*}$ and $\angle_{*}$ in terms of $P_{m-1}$ and $\bar{P}_{m-1}$, respectively. The indifference relations $I_{P}$, corresponding to $P=P_{m-1}$ and $P=\bar{P}_{m-1}$, are of the (same) form: $(x, y) \in I_{P}$ iff $v(x)=v(y)$ iff $\bar{v}(x)=\bar{v}(y)$, i.e., taking into account (2), $(x, y) \in I_{P}$ iff $v_{j}(x)=v_{j}(y)$ for all $j \in[1, m]$. This means that $(x, y) \in I_{P}$ iff $x^{*}=y^{*}$ iff $x_{*}=y_{*}$ iff vectors $x$ and $y$ can be transformed 
into each other by certain permutations of their coordinates (which may be interpreted as the anonymity of agents from $[1, n])$. Given $x \in X$, let $[x]_{P}=\left\{y \in X:(x, y) \in I_{P}\right\}$ be the indifference (=equivalence) class of $x$, and $X / I_{P}=\left\{[x]_{P}: x \in X\right\}$ be the quotient set of $X$ with respect to $I_{P}$. Clearly, the number of elements $\left|X / I_{P}\right|$ in $X / I_{P}$ is equal to (3).

A weak order $P$ on a finite set $X$ gives rise to the canonical ranking of $X$ (Aleskerov, Bouyssou, Monjardet, 2007; Hausdorff, 2005), which we recall now. Given $Y \subset X$, let $\pi(Y)=\{y \in Y:(x, y) \notin P$ for all $x \in Y\}$ be the set of the most preferred elements from $Y$ with respect to $P$. We set $X_{1}^{\prime}=\pi(X)$. Inductively, if $k \geq 2$, and nonempty disjoint subsets $X_{1}^{\prime}, \ldots, X_{k-1}^{\prime}$ of $X$ such that $X_{1}^{\prime} \cup \ldots \cup X_{k-1}^{\prime} \neq X$ are already constructed, we put $X_{k}^{\prime}=\pi\left(X \backslash\left(X_{1}^{\prime} \cup \ldots \cup X_{k-1}^{\prime}\right)\right)$. Since $X$ is finite, there is a unique positive integer $s=s_{P}(X)$ (more precisely, $s=\left|X / I_{P}\right|$ ) such that $X=\bigcup_{k=1}^{s} X_{k}^{\prime}$ (disjoint union). Now, setting $X_{k}=X_{s-k+1}^{\prime}$ for $k \in[1, s]$, we obtain the collection $\left\{X_{k}: k \in[1, s]\right\}=X / I_{P}$ of indifference classes (called the canonical ranking of $X)$, partitioning $X$, which characterizes $P$ as follows: given $x, y \in X$, $(x, y) \in P$ iff $x \in X_{l}$ and $y \in X_{k}$ for some $k, l \in[1, s]$ with $k<l$. In other words, $x$ is preferred to $y$ with respect to $P$ iff $x$ lies in an indifference class with greater ordinal number. Clearly, $(x, y) \in I_{P}$ iff $x, y \in X_{k}$ for some $k \in[1, s]$.

Define the function $\Phi=\Phi_{P}: X \rightarrow[1, s]$ in the following manner: given $x \in X$, since $X=\bigcup_{k=1}^{s} X_{k}$ (disjoint union), we have $x \in X_{k}$ for some unique number $k=k_{x} \in[1, s]$, and so, we set $\Phi(x)=k$. Thus, $\Phi(y)=k$ for all $y \in[x]_{P}=X_{k}$, and

$$
x \in X_{\Phi(x)}=\left\{y \in X: \Phi(y)=\Phi(x)=k_{x}\right\} \text { for all } x \in X .
$$

The function $\Phi$ is well-defined, uniquely determined, and surjective (from $X$ onto $[1, s]$ ) preference function for $P$. In (Aleskerov, Chistyakov, 2013b; Chistyakov, 2009) it is called the enumerating preference function for $P$ (EPF, for short). Any other preference function $\varphi: X \rightarrow \mathbb{R}$, representing $P$ (i.e., $(x, y) \in P$ iff $\varphi(x)>\varphi(y))$, can be expressed by means of $\Phi$ as follows: there is a strictly increasing function $f:[1, s] \rightarrow \mathbb{R}$ such that $\varphi(x)=f(\Phi(x))$ for all $x \in X$.

If $J: X \rightarrow X / I_{P}$ is the canonical surjection defined by $J(x)=[x]_{P}$, $x \in X$, and $\tilde{\Phi}: X / I_{P} \rightarrow[1, s]$ is the quotient function given by $\tilde{\Phi}\left([x]_{P}\right)=\Phi(x), x \in X$, then $\Phi(x)=\tilde{\Phi}(J(x))$ for all $x \in X$. Clearly, $\tilde{\Phi}$ is a bijection between $X / I_{P}$ and $[1, s]$.

In the case when $X=[1, m]^{n}$ and $P=P_{m-1}$ or $P=\bar{P}_{m-1}$, we have (cf. (3)) $s=\left|X / I_{P}\right|=\left|X^{*}\right|=C_{n+m-1}^{m-1}$. Furthermore, the enumerating preference functions $\Phi$ for $P_{m-1}$ (corresponding to the leximin (4)) and $\bar{\Phi}$ for $\bar{P}_{m-1}$ 
(corresponding to the leximax (4)) can be given combinatorially in terms of binomial coefficients as presented in Theorem 1 below (cf. (Aleskerov, Chistyakov, 2013b, Theorems 3.1 and 6.1)). To formulate it, we adopt the convention that $C_{k}^{k+1}=0$ for all integers $k \geq 0$ and $C_{0}^{0}=1$, and set

$$
V_{j}(x)=v_{1}(x)+\ldots+v_{j}(x) \text { for all } j \in[1, m] \text { and } x \in[1, m]^{n} .
$$

\section{Theorem 1.}

A. The EPF $\Phi: X=[1, m]^{n} \rightarrow\left[1, C_{n+m-1}^{m-1}\right]$ for $P_{m-1}$ is given by

$$
\Phi(x)=\sum_{j=1}^{m-1} C_{n-V_{j}(x)+m-j-1}^{m-j}+1 \text { for all } x \in X^{2}
$$

B. The EPF $\bar{\Phi}: X=[1, m]^{n} \rightarrow\left[1, C_{n+m-1}^{m-1}\right]$ for $\bar{P}_{m-1}$ is of the form

$$
\bar{\Phi}(x)=C_{n+m-1}^{m-1}-\sum_{j=1}^{m-1} C_{V_{j}(x)+j-1}^{j} \text { for all } x \in X .^{3}
$$

By Theorem 1 (A), given a vector $x \in[1, m]^{n}$, we know the ordinal number $\ell=\Phi(x)$ of its indifference class $[x]_{P}=X_{\ell}$ under the leximin ordering $\angle_{n}^{*}$ of $[1, m]^{n}$, corresponding to the weak order $P=P_{m-1}$. In addition, if $y \in[1, m]^{n}$, we have $y L_{n}^{*} x$ iff $(x, y) \in P_{m-1}$ iff $\Phi(x)>\Phi(y)$ (by Theorem 1 (B), similar conclusions hold for the leximax ordering $\angle_{*_{n}}$ and $\left.\bar{P}_{m-1}\right)$.

The purpose of this paper is to solve the inverse problem (for $\left.P=P_{m-1}\right)$ : given $\ell \in[1, s]=\left[1, C_{n+m-1}^{m-1}\right]$, restore the indifference class $X_{\ell}$, i.e., those vectors $x \in X=[1, m]^{n}$, for which $\Phi(x)=\ell$ or, equivalently, $[x]_{P}=X_{\ell}$ (in other words, find the inverse 'value' $(\tilde{\Phi})^{-1}(\ell)=[x]_{P}$ of the bijection $\left.\tilde{\Phi}: X / I_{P} \rightarrow[1, s]\right)$. Since $[x]_{P}=\{y \in X: v(x)=v(y)\}$, the problem amounts to the evaluation of the multiplicities $v_{1}(x), \ldots, v_{m-1}(x)$, which together with equality (2) gives the nondecreasing representative $x^{*}$ of $x$ from (1).

For instance, for $m=2$ we have $C_{n+m-1}^{m-1}=n+1, P_{1}=\bar{P}_{1}$ on $X \times X$ with $X=[1,2]^{n}$, and $\Phi(x)=\bar{\Phi}(x)=v_{2}(x)+1$ for all $x \in X$. So, given $\ell \in[1, n+1]$, we find $x \in X_{\ell}$ (i.e., $\Phi(x)=\ell$ ) iff $v_{1}(x)=n-\ell+1$ and $v_{2}(x)=\ell-1$ iff $x^{*}=\left(1^{n-\ell+1}, 2^{\ell-1}\right)$. In this way, we have restored the indifference class $[x]_{P_{1}}=[x]_{\bar{P}_{1}}$ from its ordinal (or aggregate) number $\Phi(x)=\ell$ under the weak order $P_{1}=\bar{P}_{1}$ (note that $P_{m-1} \neq \bar{P}_{m-1}$ for any integer $\left.m \geq 3\right)$.

The possibility of restoring the indifference class $[x]_{P}$ via its ordinal number $\Phi(x)=\ell$ under the threshold preference order $P=P_{m-1}$ on $X=[1, m]^{n}$ in the general case $m \geq 3$ was indicated in (Aleskerov, Chistyakov, 2013b, Theorems 3.2 and 3.3), which we recall as Theorem 2.

Theorem 2. Suppose $\ell$ is a positive integer and $n_{0}=n$. We have:

a) $\ell \in\left[1, C_{n+m-1}^{m-1}\right]$ iff there is a unique collection of $m-2$ integers $n_{1}, \ldots, n_{m-2}$ satisfying $0 \leq n_{j} \leq n_{j-1}$ for all $j \in[1, m-2]$ such that $\ell \in\left[L+1, L+1+n_{m-2}\right]$, where $L=\sum_{j=1}^{m-2} C_{n_{j}+m-j-1}^{m-j}$;

\footnotetext{
${ }^{2}$ More explicitly, $\Phi(x)=\sum_{j=1}^{m-2} \frac{1}{(m-j) !} \prod_{k=0}^{m-j-1}\left(k+\sum_{i=j+1}^{m} v_{i}(x)\right)+v_{m}(x)+1$.

${ }^{3}$ More explicitly, $\bar{\Phi}(x)=C_{n+m-1}^{m-1}-\sum_{j=1}^{m-1} \frac{1}{j !} \prod_{k=0}^{j-1}\left(k+\sum_{i=1}^{j} v_{i}(x)\right)$.
} 
b) given $\ell \in\left[1, C_{n+m-1}^{m-1}\right], x \in X_{\ell}$ (i.e., $\Phi(x)=\ell$ ) iff $v_{j}(x)=n_{j-1}-n_{j}$ for all $j \in[1, m-2], v_{m-1}(x)=L+1+n_{m-2}-\ell$, and $v_{m}(x)=\ell-L-1$.

However, no explicit procedure how to evaluate numbers $n_{1}, \ldots, n_{m-2}$ via the given number $\ell$ was presented in (Aleskerov, Chistyakov, 2013b). In this paper, to evaluate these numbers, we present a rigorous recursive algorithm.

\section{Main Theorem}

Our recursive algorithm for restoring indifference classes is based on the following theorem concerning (more subtle) properties of the EPF $\Phi$ from (5).

Theorem 3. Let $k \in[0, m-2], \quad 0 \leq n_{k} \leq n_{k-1} \leq \ldots \leq n_{1} \leq n_{0}=n \quad b e$ integers, and $L_{k}=\sum_{j=1}^{k} C_{n_{j}+m-j-1}^{m-j}$ (with $\left.L_{0}=0\right)$. Given $x \in X=[1, m]^{n}$ such that $v_{j}(x)=n_{j-1}-n_{j}$ for all $j \in[1, k]$, and $i \in\left[0, n_{k}\right]$, we have

$$
v_{k+1}(x)=n_{k}-i \quad \text { iff } \Phi(x)-L_{k} \in\left[C_{i+m-k-2}^{m-k-1}+1, C_{i+m-k-1}^{m-k-1}\right] .
$$

Furthermore, if $n_{1}, \ldots, n_{m-2}$ (and so, $L_{1}, \ldots, L_{m-2}$ ) are already calculated, we get $v_{m}(x)=\Phi(x)-L_{m-2}-1$ and $v_{m-1}(x)=n_{m-2}-v_{m}(x)$.

P r o o f. We divide the proof into four steps for clarity. In the first step, we need the formula for the summation of binomial coefficients over successive lower indices (see (Graham et al., 1994, formulas (5.9) and (5.10))): given two nonnegative integers $p$ and $q$,

$$
\sum_{k=0}^{q} C_{p+k}^{p}=1+C_{p+1}^{p}+\ldots+C_{p+q}^{p}=C_{p+q+1}^{p+1}=C_{p+q+1}^{q} .
$$

Step 1. First, we show that

$$
v_{k+1}(x)<n_{k}-i \text { iff } \Phi(x)>L_{k}+C_{i+m-k-1}^{m-k-1} .
$$

Let $y \in[1, m]^{n}$ be such that its nondecreasing representative $y^{*}$ is of the form $y^{*}=\left(1^{n-n_{1}}, 2^{n_{1}-n_{2}}, \ldots, k^{n_{k-1}-n_{k}},(k+1)^{n_{k}-i}, m^{i}\right)$, i.e., $v_{j}(y)=n_{j-1}-n_{j}$ for all $j \in[1, k], v_{k+1}(y)=n_{k}-i, v_{j}(y)=0$ for all $j \in[k+2, m-1]$, and $v_{m}(y)=i$. Making use of (5), let us calculate the value $\Phi(y)$. In order to do this, note that

$$
V_{j}(y)=n-n_{j} \quad \forall j \in[1, k], \text { and } V_{j}(y)=n-i \quad \forall j \in[k+1, m-1]
$$

(and, by (2), $V_{m}(y)=n$ ). If $i=0$, we find, by (5) and (9),

$$
\Phi(y)=\sum_{j=1}^{k} C_{n-\left(n-n_{j}\right)+m-j-1}^{m-j}+\sum_{j=k+1}^{m-1} C_{n-n+m-j-1}^{m-j}+1 .
$$

In the second sum at the right, the summation index $j \in[k+1, m-1]$, which implies $m-j-1 \in[0,(m-2)-k]$, and so, $C_{m-j-1}^{m-j}=0$. It follows that

$$
\Phi(y)=\Phi\left(y^{*}\right)=\sum_{j=1}^{k} C_{n_{j}+m-j-1}^{m-j}+1=L_{k}+1 .
$$

Now, if $i \geq 1$, we have, by virtue of (5), (9), and (7), 


$$
\begin{aligned}
\Phi(y)= & \Phi\left(y^{*}\right)=\sum_{j=1}^{k} C_{n_{j}+m-j-1}^{m-j}+\sum_{j=k+1}^{m-1} C_{i+m-j-1}^{m-j}+1=L_{k}+\sum_{j=k+1}^{m-1} C_{i-1+m-j}^{i-1}+1= \\
& =L_{k}+1+\sum_{p=1}^{m-k-1} C_{i-1+p}^{i-1}=L_{k}+C_{i-1+(m-k-1)+1}^{m-k-1}=L_{k}+C_{i+m-k-1}^{m-k-1} .
\end{aligned}
$$

Since $C_{m-k-1}^{m-k-1}=1,(10)$ implies that (11) holds for $i=0$ as well.

In order to prove (8), suppose $v_{k+1}(x)<n_{k}-i$. Since $v_{j}(x)=n_{j-1}-n_{j}$ for all $j \in[1, k]$ and $v_{k+1}(x)<n_{k}-i=v_{k+1}(y)$, we find $v(x) \angle_{m-1} v(y)$, and so, $(x, y) \in P_{m-1}$ (and $y L_{n}^{*} x$, by (4)). Noting that, by Theorem 1 (A), $\Phi$ is a preference function for $P_{m-1}$, we get $\Phi(x)>\Phi(y)$. Taking into account (11), this proves the 'only if' part in (8).

To prove the 'if' part in (8), we assume that $\Phi(x)>L_{k}+C_{i+m-k-1}^{m-k-1}$, i.e., by (11), $\Phi(x)>\Phi(y)$. Theorem 1 (A) implies $(x, y) \in P_{m-1}$, and so, $v(x) \angle_{m-1} v(y)$, where the two vectors $v(x)=\left(v_{1}(x), \ldots, v_{m-1}(x)\right)$ and $v(y)=\left(v_{1}(y), \ldots, v_{m-1}(y)\right)$ are given by

$$
\begin{gathered}
v(x)=\left(n-n_{1}, n_{1}-n_{2}, \ldots, n_{k-1}-n_{k}, v_{k+1}(x), v_{k+2}(x), \ldots, v_{m-1}(x)\right), \\
v(y)=\left(n-n_{1}, n_{1}-n_{2}, \ldots, n_{k-1}-n_{k}, n_{k}-i, 0, \ldots, 0\right) \in[0, n]^{m-1} .
\end{gathered}
$$

Since $v_{j}(x)=n_{j-1}-n_{j}=v_{j}(y)$ for all $j \in[1, k]$ and $v_{j}(x) \geq 0=v_{j}(y)$ for all $j \in[k+2, m-1]$, the definition of the lexicographic order $\angle_{m-1}\left(\right.$ on $\mathbb{R}^{m-1}$ ) implies $v_{k+1}(x)<v_{k+1}(y)=n_{k}-i$. This completes the proof of $(8)$.

Step 2. In this step, we show that

$$
v_{k+1}(x)>n_{k}-i \text { iff } \Phi(x)<L_{k}+C_{i+m-k-2}^{m-k-1}+1 .
$$

Let $z \in[1, m]^{n}$ be such that its nondecreasing representative $z^{*}$ is of the form $z^{*}=\left(1^{n-n_{1}}, 2^{n_{1}-n_{2}}, \ldots, k^{n_{k-1}-n_{k}},(k+1)^{n_{k}-i},(k+2)^{i}\right)$, i.e., $v_{j}(z)=n_{j-1}-n_{j}$ for all $j \in[1, k], v_{k+1}(z)=n_{k}-i, v_{k+2}(x)=i$, and (if $\left.k+3 \leq m\right) v_{j}(z)=0$ for all $j \in[k+3, m]$. Let us evaluate $\Phi(z)$ by means of (5). Since $V_{j}(z)=n-n_{j}$ for all $j \in[1, k], V_{k+1}(x)=n-i$, and $V_{j}(z)=n$ for all $j \in[k+2, m]$, applying (5), we get

$$
\Phi(z)=\sum_{j=1}^{k} C_{n_{j}+m-j-1}^{m-j}+C_{n-(n-i)+m-(k+1)-1}^{m-(k+1)}+\sum_{j=k+2}^{m-1} C_{m-j-1}^{m-j}+1 .
$$

In the third term at the right, the summation index $j \in[k+2, m-1]$, which implies $m-j-1 \in[0,(m-3)-k]$, and so, $C_{m-j-1}^{m-j}=0$. It follows that

$$
\Phi(z)=\Phi\left(z^{*}\right)=L_{k}+C_{i+m-k-2}^{m-k-1}+1 .
$$

To prove (13), suppose $v_{k+1}(x)>n_{k}-i$. Since $v_{j}(z)=n_{j-1}-n_{j}=v_{j}(x)$ for all $j \in[1, k]$ and $v_{k+1}(z)=n_{k}-i<v_{k+1}(x)$, we find $v(z) \angle_{m-1} v(x)$, and so, $(z, x) \in P_{m-1}$. By Theorem 1 (A), $\Phi$ is a preference function for $P_{m-1}$, and so, $\Phi(z)>\Phi(x)$, which together with (14) proves implication $(\Rightarrow)$ in (13).

In order to prove implication $(\Leftarrow)$ in $(13)$, we assume that $\Phi(x)<\Phi(z)$, where $\Phi(z)$ is given by (14). Applying Theorem 1 (A), we find $(z, x) \in P_{m-1}$, and so, $v(z) \angle_{m-1} v(x)$, where $v(x)$ is given by (12) and $v(z) \in[0, n]^{m-1}-$ by $v(z)=\left(n-n_{1}, n_{1}-n_{2}, \ldots, n_{k-1}-n_{k}, n_{k}-i, i, 0, \ldots, 0\right)$. Since $v_{j}(z)=n_{j-1}-n_{j}=v_{j}(x)$ for all $j \in[1, k]$, the definition of $v(z) \angle_{m-1} v(x)$ implies that either 
a) $n_{k}-i=v_{k+1}(z)<v_{k+1}(x)$, or

b) (if $k+3 \leq m$ ) $n_{k}-i=v_{k+1}(x)$ and $i=v_{k+2}(z)<v_{k+2}(x)$, or

c) (if $k+4 \leq m) n_{k}-i=v_{k+1}(x), i=v_{k+2}(x)$ and $0=v_{k+3}(z)<v_{k+3}(x)$, or

d) (if $k+5 \leq m) n_{k}-i=v_{k+1}(x), i=v_{k+2}(x)$, and there is $p \in[k+4, m-1]$ such that $0=v_{j}(z)=v_{j}(x)$ for all $j \in[k+3, p-1]$ and $0=v_{p}(z)<v_{p}(x)$.

Let us show that possibilities b), c), and d) cannot hold. In fact, if any one of the three possibilities holds, then $n_{k}=v_{k+1}(x)+i$, and so, by (2),

we get
$v_{k+1}(x)+\ldots+v_{m}(x)=n-\sum_{j=1}^{k} v_{j}(x)=n-\sum_{j=1}^{k}\left(n_{j-1}-n_{j}\right)=n-\left(n-n_{k}\right)=n_{k}=v_{k+1}(x)+i$, whence $i=v_{k+2}(x)+\ldots+v_{m}(x)$. It follows that $i \geq v_{k+2}(x)$, and so, b) is impossible. Furthermore, if $i=v_{k+2}(x)$, then $v_{j}(x)=0$ for all $j \in[k+3, m]$, which implies that c) and d) cannot hold as well. Thus, only possibility a) above holds, which proves implication $(\Leftarrow)$ in $(13)$.

Step 3. Assertion (8) implies $v_{k+1}(x) \geq n_{k}-i$ iff $\Phi(x)-L_{k} \leq C_{i+m-k-1}^{m-k-1}$, and assertion (13) implies $v_{k+1}(x) \leq n_{k}-i$ iff $\Phi(x)-L_{k} \geq C_{i+m-k-2}^{m-k-1}+1$. This completes the proof of assertion (6).

Step 4. Now suppose that, by means of (6), the numbers $n_{1}, \ldots, n_{m-2}$ are already calculated, which corresponds to the case $k=m-2$. Noting that, for all $j \in[1, m-2]$, we have $v_{j}(x)=n_{j-1}-n_{j}$ and, by (2),

$$
n-V_{j}(x)=n-\sum_{i=1}^{j} v_{i}(x)=n-\sum_{i=1}^{j}\left(n_{i-1}-n_{i}\right)=n-\left(n_{0}-n_{j}\right)=n_{j},
$$

by virtue of (5), we get $\Phi(x)=\sum_{j=1}^{m-2} C_{n_{j}+m-j-1}^{m-j}+v_{m}(x)+1=L_{m-2}+v_{m}(x)+1$. Since the value of $L_{m-2}$ is already known, we find

$$
v_{m}(x)=\Phi(x)-L_{m-2}-1 .
$$

On the other hand, putting $k=m-2$ in (6), we get $m-k-1=m-(m-2)-1=1$, and so, assertion (6) reads as follows: given $i \in\left[0, n_{m-2}\right]$, we have

$$
\begin{aligned}
v_{m-1}(x) & =n_{m-2}-i \text { iff } \Phi(x)-L_{m-2} \in\left[C_{i}^{1}+1, C_{i+1}^{1}\right]= \\
& =[i+1, i+1] \text { iff } \Phi(x)-L_{m-2}=i+1 .
\end{aligned}
$$

From here and (16), we conclude that

$$
v_{m-1}(x)=n_{m-2}-i=n_{m-2}-\left(\Phi(x)-L_{m-2}-1\right)=n_{m-2}-v_{m}(x) .
$$

This completes the proof of Theorem 3 .

Remark. Theorem 3 applies in the particular case when $m=2$ as well: in fact, we have $k=0, L_{k}=L_{0}=0$, and, for $i \in\left[0, n_{0}\right]=[0, n]$, assertion (6) means that (given $\left.x \in[1,2]^{n}\right) \quad v_{1}(x)=n-i$ iff $\Phi(x) \in\left[C_{i}^{1}+1, C_{i+1}^{1}\right]=\{i+1\}$ iff $\Phi(x)=i+1$, whence $\Phi(x)=\left(n-v_{1}(x)\right)+1=v_{2}(x)+1$. Hence, if $\ell=\Phi(x)$, then $i=\ell-1, \quad v_{1}(x)=n-i=n-\ell+1$ and $v_{2}(x)=\ell-1$, i.e., $x^{*}=\left(1^{n-\ell+1}, 2^{\ell-1}\right)$, $\ell \in[0, n]$. 
Remark. The quantities $v_{m-1}(x)$ and $v_{m}(x)$ in Theorem 3 and Theorem 2 are coherent (=the same) in the sense that, by (18), $v_{m-1}(x)=L+1+n_{m-2}-\ell$ and, by $(16), v_{m}(x)=\ell-L-1$, where $L=L_{m-2}$ and $\ell=\Phi(x)$.

\section{Algorithm for Restoring Indifference Classes}

Let $n \geq 2$ and $m \geq 3$ be integers, $X=[1, m]^{n}$, and $P=P_{m-1}$ be the threshold preference order on $X$ (corresponding to the leximin ordering $\angle_{n}^{*}$ ). Given a number $\ell \in[1, s]=\left[1, C_{n+m-1}^{m-1}\right]$, we are looking for members $x$ from the indifference class $X_{\ell}=\{x \in X: \Phi(x)=\ell\}$ (the EPF $\Phi$ for $P_{m-1}$ given by (5)), which is equivalent to finding multiplicities $v_{1}(x), \ldots, v_{m}(x)$ in the nondecreasing representative (1) of $x \in X_{\ell}$.

The idea of the recursive algorithm, restoring $x \in X_{\ell}$, lies in the fact that the interval $\left[1, C_{n+m-1}^{m-1}\right]$ can be decomposed into the following disjoint union of (adjacent) natural intervals:

$$
\begin{aligned}
& {\left[1, C_{n+m-1}^{m-1}\right]=\bigcup_{i=0}^{n}\left[C_{i+m-2}^{m-1}+1, C_{i+m-1}^{m-1}\right]=} \\
& =\bigcup_{i=0}^{n}\left[C_{i+m-2}^{m-1}+1, C_{i+m-2}^{m-1}+C_{i+m-2}^{m-2}\right] .
\end{aligned}
$$

We will apply the following 'cyclic procedure'. Suppose numbers $n, m, L$ and $\ell$ are such that

$$
\ell-L \in\left[1, C_{n+m-1}^{m-1}\right] .
$$

We set $\ell^{\prime}=\ell-L$. By (21) and (19), there is a unique number $n^{\prime} \in[0, n]$ such that

$$
\ell^{\prime} \in\left[C_{n^{\prime}+m-2}^{m-1}+1, C_{n^{\prime}+m-1}^{m-1}\right]=\left[C_{n^{\prime}+m-2}^{m-1}+1, C_{n^{\prime}+m-2}^{m-1}+C_{n^{\prime}+(m-1)-1}^{(m-1)-1}\right] .
$$

Now we set $m^{\prime}=m-1$ and $L^{\prime}=C_{n^{\prime}+m-2}^{m-1}=C_{n^{\prime}+m^{\prime}-1}^{m^{\prime}}$. By (22), we find that $\ell^{\prime} \in\left[L^{\prime}+1, L^{\prime}+C_{n^{\prime}+m^{\prime}-1}^{m^{\prime}-1}\right]$, and so, $\ell^{\prime}-L^{\prime} \in\left[1, C_{n^{\prime}+m^{\prime}-1}^{m^{\prime}-1}\right]$.

Having obtained (21) for the new (primed) numbers $n^{\prime}, m^{\prime}, L^{\prime}$ and $\ell^{\prime}$, we reduced the dimension (at least in $m$ ), and may apply the procedure one more time. In order to evaluate the multiplicities $v_{j}(x)$, in what follows we combine the cyclic procedure with Theorem 3 by introducing nonnegative integers $j$ and defining numbers $n=n_{j}, m=m_{j}, L=L_{j}^{\prime}$ and $\ell=\ell_{j-1}$ and their primed counterparts $n^{\prime}=n_{j+1}, m^{\prime}=m_{j+1}, L^{\prime}=C_{n^{\prime}+m^{\prime}-1}^{m^{\prime}}$ and $\ell^{\prime}=\ell_{j}$ appropriately.

Before we turn to the general algorithm, for the sake of clarity we first present the initial part of it (having its own additional details) when the first three multiplicities $v_{1}(x), v_{2}(x)$, and $v_{3}(x)$ are evaluated. Note that the 'nonprimed' numbers $L_{0}, \ldots, L_{m-2}$, to be used below, are exactly the numbers from Theorem 3 .

\subsection{Evaluation of $v_{1}(x)$}

We set $n_{0}=n, m_{0}=m, L_{0}=0$, and $\ell_{0}=\ell$, where $\ell=\Phi(x) \in\left[1, C_{n+m-1}^{m-1}\right]$. 
Since $\ell_{0} \in\left[1, C_{n+m-1}^{m-1}\right],(19)$ implies the existence of a unique $n_{1} \in[0, n]$ such that

$$
\ell_{0} \in\left[C_{n_{1}+m-2}^{m-1}+1, C_{n_{1}+m-1}^{m-1}\right]=\left[C_{n_{1}+m-2}^{m-1}+1, C_{n_{1}+m-2}^{m-1}+C_{n_{1}+(m-1)-1}^{(m-1)-1}\right] .
$$

Noting that the first interval here coincides with the interval from (6) with $k=0$ and $i=n_{1}$, and $\ell_{0}=\Phi(x)-L_{0}$, by Theorem 3 (with $k=0$ and $i=n_{1}$ ), we find

$$
v_{1}(x)=n_{0}-n_{1}=n-n_{1} .
$$

If $n_{1}=0$, then, by virtue of (2) (and (23)), $v_{2}(x)=v_{3}(x)=\ldots=v_{m}(x)=0$.

4.2. Evaluation of $v_{2}(x)$

Now, knowing $1 \leq n_{1} \leq n$, we set $m_{1}=m_{0}-1, L_{1}^{\prime}=L_{1}=C_{n_{1}+m-2}^{m-1}=C_{n_{1}+m_{1}-1}^{m_{1}}$, and $\ell_{1}=\ell_{0}-L_{1}^{\prime}$. By (23), $\ell_{0} \in\left[L_{1}^{\prime}+1, L_{1}^{\prime}+C_{n_{1}+m_{1}-1}^{m_{1}-1}\right]$, and so, $\ell_{1} \in\left[1, C_{n_{1}+m_{1}-1}^{m_{1}-1}\right]$. From (19) (with $n$ replaced by $n_{1}$ and $m-$ by $m_{1}$ ), we obtain a unique number $n_{2} \in\left[0, n_{1}\right]$ such that

$$
\ell_{1} \in\left[C_{n_{2}+m_{1}-2}^{m_{1}-1}+1, C_{n_{2}+m_{1}-1}^{m_{1}-1}\right]=\left[C_{n_{2}+m-3}^{m-2}+1, C_{n_{2}+m-3}^{m-2}+C_{n_{2}+\left(m_{1}-1\right)-1}^{\left(m_{1}-1\right)-1}\right] .
$$

The first interval here is of the form $\left[C_{n_{2}+m-1-2}^{m-1-1}+1, C_{n_{2}+m-1-1}^{m-1-1}\right]$ and coincides with the interval from (6) with $k=1$ and $i=n_{2}$, equality (24) holds with $1 \leq n_{1} \leq n_{0}=n$, and $\ell_{1}=\Phi(x)-L_{1}$. By Theorem 3 (with $k=1$ and $i=n_{2}$ ), we get

$$
v_{2}(x)=n_{1}-n_{2} .
$$

If $n_{2}=0$, then by (2) and (24), $v_{3}(x)=v_{4}(x)=\ldots=v_{m}(x)=0$.

\subsection{Evaluation of $v_{3}(x)$}

Knowing $1 \leq n_{2} \leq n_{1}$, we set $m_{2}=m_{1}-1=m-2, L_{2}^{\prime}=C_{n_{2}+m-3}^{m-2}=C_{n_{2}+m_{2}-1}^{m_{2}}$, and $\ell_{2}=\ell_{1}-L_{2}^{\prime}$. By (25), $\ell_{1} \in\left[L_{2}^{\prime}+1, L_{2}^{\prime}+C_{n_{2}+m_{2}-1}^{m_{2}-1}\right]$, and so, $\ell_{2} \in\left[1, C_{n_{2}+m_{2}-1}^{m_{2}-1}\right]$. By (19) (with $n$ replaced by $n_{2}$ and $m-$ by $m_{2}$ ), there is a unique number $n_{3}$ from $\left[0, n_{2}\right]$ such that

$$
\ell_{2} \in\left[C_{n_{3}+m_{2}-2}^{m_{2}-1}+1, C_{n_{3}+m_{2}-1}^{m_{2}-1}\right]=\left[C_{n_{3}+m-4}^{m-3}+1, C_{n_{3}+m-4}^{m-3}+C_{n_{3}+\left(m_{2}-1\right)-1}^{\left(m_{2}-1\right)-1}\right] .
$$

The first interval here is of the form $\left[C_{n_{3}+m-2-2}^{m-2-1}+1, C_{n_{3}+m-2-1}^{m-2-1}\right]$ and coincides with the interval from (6) with $k=2$ and $i=n_{3}, 1 \leq n_{2} \leq n_{1} \leq n_{0}=n$, equalities (24) and (26) hold, and $\ell_{2}=\ell_{1}-L_{2}^{\prime}=\left(\ell_{0}-L_{1}^{\prime}\right)-L^{\prime}=$ $=\ell_{0}-C_{n_{1}+m-1-1}^{m-1}-C_{n_{2}+m-2-1}^{m-2}=\Phi(x)-L_{2}$. By Theorem 3 (with $k=2$ and $\left.i=n_{3}\right)$, we find $v_{3}(x)=n_{2}-n_{3}$. If $n_{3}=0$, then by (2), (24), and (26), $v_{4}(x)=v_{5}(x)=\ldots=v_{m}(x)=0$.

Now, we are in a position to present the general algorithm.

4.4. Recursive Algorithm for Restoring $v_{1}(x), \ldots, v_{m}(x)$

The initial input data (for $j=0$ ) is as follows:

$$
n_{0}=n, m_{0}=m, L_{-1}=L_{0}=0 \text {, and } \ell_{-1}=\ell \text {, where } \ell=\Phi(x) \in\left[1, C_{n+m-1}^{m-1}\right] \text {. }
$$


Assume that, for $j \in[0, m-3]$, we already know integers $0 \leq n_{j} \leq n_{j-1} \leq \ldots \leq n_{1} \leq n_{0}=n, m_{j}=m-j, \quad L_{0}^{\prime}=0, \quad L_{j}^{\prime}=C_{n_{j}+m_{j}-1}^{m_{j}}$ if $j \geq 1$, and $\ell_{j-1}=\Phi(x)-L_{j-1}$ such that $\ell_{j-1}-L_{j}^{\prime} \in\left[1, C_{n_{j}+m_{j}-1}^{m_{j}-1}\right]$, and we know that

$$
v_{1}(x)=n-n_{1}, v_{2}(x)=n_{1}-n_{2}, \ldots, \quad v_{j}(x)=n_{j-1}-n_{j}
$$

(the previous line being treated as empty if $j=0$ ). In Sections $4.1-4.3$, the validity of this assumption is explicitly verified for $j=0,1,2$, respectively.

We set $\ell_{j}=\ell_{j-1}-L_{j}^{\prime}$. Since $\ell_{j} \in\left[1, C_{n_{j}+m_{j}-1}^{m_{j}-1}\right]$, by virtue of (19) (with $n$ replaced by $n_{j}$ and $m-$ by $\left.m_{j}\right)$, there exists a unique $n_{j+1} \in\left[0, n_{j}\right]$ such that $^{4}$

$$
\begin{gathered}
\ell_{j} \in\left[C_{\left(n_{j+1}\right)+m_{j}-2}^{m_{j}-1}+1, C_{\left(n_{j+1}\right)+m_{j}-1}^{m_{j}-1}\right]= \\
=\left[C_{\left(n_{j+1}\right)+\left(m_{j}-1\right)-1}^{m_{j}-1}+1, C_{\left(n_{j+1}\right)+\left(m_{j}-1\right)-1}^{m_{j}-1}+C_{\left(n_{j+1}\right)+\left(m_{j}-1\right)-1}^{\left(m_{j}-1\right)-1}\right] .
\end{gathered}
$$

The interval in (28) coincides with the interval from (6) with $k=j$ and $i=n_{j+1}$; we have equalities (27) with $0 \leq n_{j} \leq n_{j-1} \leq \ldots \leq n_{1} \leq n_{0}=n$, and

$$
\ell_{j}=\ell_{j-1}-L_{j}^{\prime}=\left(\Phi(x)-L_{j-1}\right)-C_{n_{j}+m-j-1}^{m-j}=\Phi(x)-L_{j} .
$$

By Theorem 3 (with $k=j$ and $i=n_{j+1}$ ), we get $v_{j+1}(x)=n_{j}-n_{j+1}$. If $n_{j+1}=0$, then, by (2) and (27), $v_{j+2}(x)=\ldots=v_{m}(x)=0$, so we may assume that $n_{j+1} \geq 1$. Now, we set $m_{j+1}=m_{j}-1$ and $L_{j+1}^{\prime}=C_{\left(n_{j+1}\right)+\left(m_{j+1}\right)-1}^{m_{j+1}}$ (cf. (29)), note that (30) holds and, by (29),

$$
\ell_{j}-L_{j+1}^{\prime} \in\left[1, C_{\left(n_{j+1}\right)+\left(m_{j+1}\right)-1}^{\left(m_{j+1}\right)-1}\right] .
$$

Thus, our assumption (preceding (27)) holds with $j$ replaced by $j+1$. We set $\ell_{j+1}=\ell_{j}-L_{j+1}^{\prime}$, take into account equality $\ell_{j+1}=\Phi(x)-L_{j+1}$ (which follows from (30) ), and repeat the whole procedure once again.

Putting successively $j=0,1,2, \ldots, m-3$, we obtain integers $0 \leq n_{m-2} \leq n_{m-3} \leq \ldots \leq n_{1} \leq n_{0}=n, \quad$ and so, integers $L_{1}, \ldots, L_{m-2}, \quad$ and $\ell_{1}=\ell-L_{1}, \ell_{2}=\ell-L_{2}, \ldots, \ell_{m-2}=\ell-L_{m-2}=\Phi(x)-L_{m-2}$. By Theorem 3, we conclude that

$v_{j}(x)=n_{j-1}-n_{j} \forall j \in[1, m-2], v_{m}(x)=\ell_{m-2}-1$, and $v_{m-1}(x)=n_{m-2}-v_{m}(x)$.

Let us verify that if $x \in X$ satisfies conditions on the previous line, then $\Phi(x)=\ell$. In fact, by virtue of (5) and (15), we have

$$
\begin{gathered}
\Phi(x)=\sum_{j=1}^{m-2} C_{n_{j}+m-j-1}^{m-j}+v_{m}(x)+1=L_{m-2}+\left(\ell_{m-2}-1\right)+1= \\
=L_{m-2}+\left(\ell-L_{m-2}-1\right)+1=\ell .
\end{gathered}
$$

Remark. Equalities for $v_{m-1}(x)$ and $v_{m}(x)$ may also be written in the form $v_{m-1}(x)=n_{m-2}-n_{m-1}$ and $v_{m}(x)=n_{m-1}-n_{m}$. It suffices to denote $i \in\left[0, n_{m-2}\right]$ in (17) by $i=n_{m-1}$ and note that, by (16), $n_{m-1}=v_{m}(x)$, and so, $n_{m}=0$.

Let us illustrate the above algorithm by an example (with small dimensions).

\footnotetext{
${ }^{4}$ For the sake of better (nonambiguous) readability, number $n_{j+1}$ is written as $\left(n_{j+1}\right)$.
} 
Example 1. Let $n=11$ and $m=6$, so that $X=[1,6]^{11}$ and $s=\left|X^{*}\right|=C_{n+m-1}^{m-1}=C_{16}^{5}=4368$. We are going to restore the indifference class $X_{2202}$ under $P=P_{m-1}=P_{5}$ (corresponding to $\left.\angle_{11}^{*}\right)$, i.e., $x=\left(x_{1}, \ldots, x_{11}\right) \in X$ such that $x \in X_{2202}$ (or $\Phi(x)=\ell$ with $\ell=2202$ ). For this, we evaluate the quantities $v_{1}(x), \ldots, v_{6}(x)$.

Since intervals at the right-hand side in decomposition (19) are adjacent, we calculate only the right endpoints $C_{i+m-1}^{m-1}$ for $i=0, \ldots, n$, noting that $C_{m-1}^{m-1}=1$ (for $\left.i=0\right)$ and $C_{m}^{m-1}=m$ (for $i=1$ ).

Step 1. Set $n=11, m=6$, and $\ell_{0}=2202 \in[1,4368]$. The right endpoints are of the form $C_{i+m-1}^{m-1}=C_{i+5}^{5}, i=0, \ldots, 11$, and assume the values ${ }^{5}$ :

\begin{tabular}{|c|c|c|c|c|c|c|c|c|c|c|c|c|}
\hline$i$ & 0 & 1 & 2 & 3 & 4 & 5 & 6 & 7 & 8 & 9 & $\mathbf{1 0}$ & 11 \\
\hline$C_{i+5}^{5}$ & 1 & 6 & 21 & 56 & 126 & 252 & 462 & 792 & 1287 & $\mathbf{2 0 0 2}$ & 3003 & 4368 \\
\hline
\end{tabular}

It is seen from the table that $\ell_{0}=2202$ belongs to $[2002+1,3003]$, which corresponds to $i=10$. Thus, $L_{1}^{\prime}=2002, n_{1}=10$, and $v_{1}(x)=n-n_{1}=$ $=11-10=1$.

Step 2. Set $n=n_{1}=10, m=6-1=5$, and $\ell_{1}=\ell_{0}-L_{1}^{\prime}=2202-2002=$ $=200$, so that $\ell_{1} \in[1,1001]$. The right endpoints $C_{i+m-1}^{m-1}=C_{i+4}^{4}, i \in[0,10]$, are given by

\begin{tabular}{|c|c|c|c|c|c|c|c|c|c|c|c|}
\hline$i$ & 0 & 1 & 2 & 3 & 4 & 5 & $\mathbf{6}$ & 7 & 8 & 9 & 10 \\
\hline$C_{i+4}^{4}$ & 1 & 5 & 15 & 35 & 70 & $\mathbf{1 2 6}$ & 210 & 330 & 495 & 715 & 1001 \\
\hline
\end{tabular}

Number $\ell_{1}=200$ belongs to the interval $[126+1,210]$, which corresponds to $i=6$. It follows that $L_{2}^{\prime}=126, n_{2}=6$, and $v_{2}(x)=n_{1}-n_{2}=10-6=4$.

Step 3. Set $n=n_{2}=6, m=5-1=4$, and $\ell_{2}=\ell_{1}-L_{2}^{\prime}=200-126=74$, and so, $\ell_{2} \in[1,84]$. The right endpoints $C_{i+m-1}^{m-1}=C_{i+3}^{3}, i \in[0,6]$, are as follows:

\begin{tabular}{|c|c|c|c|c|c|c|c|}
\hline$i$ & 0 & 1 & 2 & 3 & 4 & 5 & $\mathbf{6}$ \\
\hline$C_{i+3}^{3}$ & 1 & 4 & 10 & 20 & 35 & $\mathbf{5 6}$ & 84 \\
\hline
\end{tabular}

Number $\ell_{2}=74$ belongs to the interval $[56+1,84]$, which corresponds to $i=6$. Hence, $L_{3}^{\prime}=56, n_{3}=6$, and $v_{3}(x)=n_{2}-n_{3}=6-6=0$.

Step 4. Set $n=n_{3}=6, m=4-1=3$, and $\ell_{3}=\ell_{2}-L_{3}^{\prime}=74-56=18$, which implies $\ell_{3} \in[1,28]$. The right endpoints $C_{i+m-1}^{m-1}=C_{i+2}^{2}, i \in[0,6]$, are given by

\begin{tabular}{|c|c|c|c|c|c|c|c|}
\hline$i$ & 0 & 1 & 2 & 3 & 4 & $\mathbf{5}$ & 6 \\
\hline$C_{i+2}^{2}$ & 1 & 3 & 6 & 10 & $\mathbf{1 5}$ & 21 & 28 \\
\hline
\end{tabular}

Number $\ell_{3}=18$ belongs to the interval $[15+1,21]$, which corresponds to $i=5$. Hence, $L_{4}^{\prime}=15, n_{4}=5$, and $v_{4}(x)=n_{3}-n_{4}=6-5=1$.

\footnotetext{
${ }^{5}$ In computing binomial coefficients, the reduction formula $C_{n+1}^{k}=(n+1) C_{n}^{k} /(n+1-k), k \in[0, n]$, is efficient. For instance, if $i=6$ (see the table), we have $C_{11}^{5}=(11 / 6) C_{10}^{5}=11 \times 252 / 6=462$.
} 
Step 5. Set $\ell_{4}=\ell_{3}-L_{4}^{\prime}=18-15=3$. Noting that $\ell_{4}=\ell_{m-2}$ and $n_{4}=n_{m-2}$ with the initial value $m=6$, we conclude that $v_{6}(x)=\ell_{4}-1=3-1=2$, and $v_{5}(x)=n_{4}-v_{6}(x)=5-2=3$.

Thus, $v_{1}(x)=1, v_{2}(x)=4, v_{3}(x)=0, v_{4}(x)=1, v_{5}(x)=3$, and $v_{6}(x)=2$, i.e., $x^{*}=\left(1^{1}, 2^{4}, 3^{0}, 4^{1}, 5^{3}, 6^{2}\right)=(1,2,2,2,2,4,5,5,5,6,6)$. Finally, let us calculate the value $\Phi(x)$ by means of (5). Since $V_{1}(x)=1, V_{2}(x)=5, V_{3}(x)=5, V_{4}(x)=6$, $V_{5}(x)=9\left(\right.$ and $\left.V_{6}(x)=11\right)$, we get (cf. $\left.(31)\right)$

$$
\begin{gathered}
\Phi(x)=\sum_{j=1}^{5} C_{11-V_{j}(x)+6-j-1}^{6-j}+1=C_{14}^{5}+C_{9}^{4}+C_{8}^{3}+C_{6}^{2}+C_{2}^{1}+1= \\
=L_{1}^{\prime}+L_{2}^{\prime}+L_{3}^{\prime}+L_{4}^{\prime}+v_{6}(x)+1=2002+126+56+15+2+1=2202 .
\end{gathered}
$$

\section{Restoring Indifference Classes for the Leximax}

The restoring of indifference classes via their ordinal numbers under the leximax preference ordering $\angle_{*_{n}}$ of $X=[1, m]^{n}$ (corresponding to the dual threshold preference order $P=\bar{P}_{m-1}$ ) can be reduced to the case of $\angle_{n}^{*}$ (and $\left.P=P_{m-1}\right)$.

Let $r:[1, m] \rightarrow[1, m]$ be the function given by $r(j)=m-j+1, j \in[1, m]$ (this function 'reverses' the natural order of $[1, m]$ ). Define the vector-function $\mathbf{r}: X=[1, m]^{n} \rightarrow X$ by $\mathbf{r}(x)=\left(r\left(x_{1}\right), \ldots, r\left(x_{n}\right)\right), x=\left(x_{1}, \ldots, x_{n}\right) \in X$. Clearly, $r(r(j))=j$ for all $j \in[1, m]$, and $\mathbf{r}(\mathbf{r}(x))=x$ for all $x \in X$. Furthermore (Aleskerov, Chistyakov, 2013b; Aleskerov et al., 2010b), given $j \in[1, m]$ and $x \in X$, we have

$$
v_{j}(\mathbf{r}(x))=v_{r(j)}(x)
$$

$\bar{v}(x)=v(\mathbf{r}(x)), \mathbf{r}\left(x^{*}\right)=(\mathbf{r}(x))_{*}$, and $\mathbf{r}\left(x_{*}\right)=(\mathbf{r}(x))^{*}$.

Suppose $\bar{\ell} \in\left[1, C_{n+m-1}^{m-1}\right]$ is given (note that $s \equiv s_{P}(X)=\left|X_{*}\right|=C_{n+m-1}^{m-1}$ if $\left.P=\bar{P}_{m-1}\right)$. In order to restore the class $X_{\bar{\ell}}$ (i.e., $x \in X$ such that $\bar{\Phi}(x)=\bar{\ell}$ with $\bar{\Phi}$ given by Theorem 1 (B)), we make use of the following observation. Since, for $j \in[1, m]$ and $x \in X$ (cf. (32)),

$$
V_{j}(\mathbf{r}(x))=\sum_{i=1}^{j} v_{i}(\mathbf{r}(x))=\sum_{i=1}^{j} v_{r(i)}(x)=\sum_{i=1}^{j} v_{m-i+1}(x)=\sum_{k=m-j+1}^{m} v_{k}(x),
$$

we have, by (2), $n-V_{j}(\mathbf{r}(x))=V_{m-j}(x)$, and so, (5) and Theorem 1 (B) imply

$$
\begin{gathered}
\Phi(\mathbf{r}(x))=\sum_{j=1}^{m-1} C_{V_{m-j}(x)+m-j-1}^{m-j}+1=\sum_{i=1}^{m-1} C_{V_{i}(x)+i-1}^{i}+1= \\
=C_{n+m-1}^{m-1}-\bar{\Phi}(x)+1=C_{n+m-1}^{m-1}-\bar{\ell}+1 .
\end{gathered}
$$

We set $\ell=C_{n+m-1}^{m-1}-\bar{\ell}+1$. Since $\bar{\ell} \in\left[1, C_{n+m-1}^{m-1}\right]$, we find, from (33),

$$
\Phi(\mathbf{r}(x))=\ell \in\left[1, C_{n+m-1}^{m-1}\right] .
$$

Making use of the algorithm from Section 4.4 and taking into account (34), we restore the values (32) for all $j=1, \ldots, m$ (i.e., $v_{m}(x), \ldots, v_{1}(x)$ ). 
Let us illustrate this procedure by an example.

Example 2. As in Example 1, we assume that $n=11, m=6, X=[1,6]^{11}$, and so, $s=\left|X_{*}\right|=C_{n+m-1}^{m-1}=C_{16}^{5}=4368$. Let us restore the indifference class $X_{2202}$ under $P=\bar{P}_{m-1}=\bar{P}_{5}$ (corresponding to the leximax ordering $\angle_{*_{11}}$ ), i.e., $x=\left(x_{1}, x_{2}, \ldots, x_{11}\right) \in X$ such that $\bar{\Phi}(x)=\bar{\ell}$ with $\bar{\ell}=2202$. In order to do this, we evaluate the quantities $v_{6}(x)=v_{r(1)}(x), \ldots, v_{1}(x)=v_{r(6)}(x)$.

We set $\ell=C_{n+m-1}^{m-1}-\bar{\ell}+1=4368-2202+1=2167$, and $r(j)=7-j$ if $j \in[1,6]$. As in Example 1, we calculate only the right endpoints $C_{i+m-1}^{m-1}$ in the decomposition (19) for $i=0, \ldots, n$, whose values, for the first four steps below, are presented in the corresponding tables in Example 1.

Step 1. Set $n=11, m=6$, and $\ell_{0}=2167 \in[1,4368]$. Since $\ell_{0} \in[2002+1,3003]$ (which corresponds to $i=10$ ), we have $L_{1}^{\prime}=2002$, $n_{1}=10$, and, by virtue of (32), $v_{6}(x)=v_{r(1)}(x)=v_{1}(\mathbf{r}(x))=n-n_{1}=11-10=1$.

Step 2. Set $n=n_{1}=10, m=5$, and $\ell_{1}=\ell_{0}-L_{1}^{\prime}=2167-2002=165$, so that $\ell_{1} \in[126+1,210]$ (corresponding to $i=6$ ). We find $L_{2}^{\prime}=126, n_{2}=6$, and, by $(32), v_{5}(x)=v_{r(2)}(x)=n_{1}-n_{2}=10-6=4$.

Step 3. Set $n=n_{2}=6, m=4$, and $\ell_{2}=\ell_{1}-L_{2}^{\prime}=165-126=39$, and so, $\ell_{2} \in[35+1,56]$ (which corresponds to $i=5$ ). Hence, $L_{3}^{\prime}=35, n_{3}=5$, and, by (32), $v_{4}(x)=v_{r(3)}(x)=n_{2}-n_{3}=6-5=1$.

Step 4. Set $n=n_{3}=5, m=3$, and $\ell_{3}=\ell_{2}-L_{3}^{\prime}=39-35=4$. We have $\ell_{3} \in[3+1,6]$ (which corresponds to $i=2$ ), and so, $L_{4}^{\prime}=3, n_{4}=2$, and, by (32), $v_{3}(x)=v_{r(4)}(x)=n_{3}-n_{4}=5-2=3$.

Step 5. Set $\ell_{4}=\ell_{3}-L_{4}^{\prime}=4-3=1$. Noting that $\ell_{4}=\ell_{m-2}$ and $n_{4}=n_{m-2}$ with the initialvalue $m=6$, we conclude that $v_{1}(x)=v_{r(6)}(x)=\ell_{4}-1=0$, and $v_{2}(x)=n_{4}-v_{r(6)}(x)=n_{4}-v_{1}(x)=2$.

Thus, $\quad v_{1}(x)=0, \quad v_{2}(x)=2, \quad v_{3}(x)=3, \quad v_{4}(x)=1, \quad v_{5}(x)=4, \quad$ and $v_{6}(x)=1$, i.e., $x^{*}=\left(1^{0}, 2^{2}, 3^{3}, 4^{1}, 5^{4}, 6^{1}\right)=(2,2,3,3,3,4,5,5,5,5,6)$. Let us calculate the value $\bar{\Phi}(x)$ by means of formula in Theorem 1 (B). Since $V_{1}(x)=0$, $V_{2}(x)=2, V_{3}(x)=5, V_{4}(x)=6, V_{5}(x)=10$ (and $V_{6}(x)=11$ ), we get

$$
\begin{gathered}
\bar{\Phi}(x)=C_{11+6-1}^{6-1}-\sum_{j=1}^{5} C_{V_{j}(x)+j-1}^{j}=C_{16}^{5}-C_{0}^{1}-C_{3}^{2}-C_{7}^{3}-C_{9}^{4}-C_{14}^{5}= \\
=4368-v_{1}(x)-L_{4}^{\prime}-L_{3}^{\prime}-L_{2}^{\prime}-L_{1}^{\prime}=4368-0-3-35-126-2002=2202 .
\end{gathered}
$$

Remark. From (34) and (19), there is a unique $i \in[0, n]$ such that $C_{i+m-2}^{m-1}+1 \leq \Phi(\mathbf{r}(x)) \leq C_{i+m-1}^{m-1}$, and so, by (33), we get $C_{n+m-1}^{m-1}-C_{i+m-1}^{m-1}+1 \leq \bar{\Phi}(x)=$ $=\bar{\ell} \leq C_{n+m-1}^{m-1}-C_{i+m-2}^{m-1}$. This gives rise to an idea of the following decomposition of the interval $\left[1, C_{n+m-1}^{m-1}\right]$ into the disjoint union of (adjacent) natural intervals:

$$
\left[1, C_{n+m-1}^{m-1}\right]=\bigcup_{i=0}^{n}\left[C_{n+m-1}^{m-1}-C_{i+m-1}^{m-1}+1, C_{n+m-1}^{m-1}-C_{i+m-2}^{m-1}\right]
$$


Applied in place of (19), this decomposition may be used to develop an alternative recursive algorithm for restoring $v_{1}(x), \ldots, v_{m}(x)$ in the case of the leximax ordering $\angle_{*_{n}}$ of $X=[1, m]^{n}$; then, the case of the leximin ordering $\angle_{n}^{*}$ of $X$ can be reduced to the former case by means of (33) written as $\bar{\Phi}(\mathbf{r}(x))=C_{n+m-1}^{m-1}-\ell+1$ with $\ell=\Phi(x) \in\left[1, C_{n+m-1}^{m-1}\right]$. We omit further details.

\section{REFERENCES}

Aleskerov F., Bouyssou D., Monjardet B. (2007). Utility Maximization, Choice and Preference. Berlin: Springer-Verlag.

Aleskerov F., Chistyakov V.V., Kalyagin V. (2010a). The Threshold Aggregation. Economics Letters, 107, 2, 261-262.

Aleskerov F., Yakuba V., Yuzbashev D. (2007). A ‘Threshold Aggregation' of ThreeGraded Rankings. Mathematical Social Sciences, 53, 1, 106-110.

Aleskerov F.T., Chistyakov V.V. (2013a). The Threshold Decision Making. Procedia Computer Science, 17, 1103-1106.

Aleskerov F.T., Chistyakov V.V. (2013b). The Threshold Decision Making Effectuated by the Enumerating Preference Function. International Journal of Information Technology and Decision Making, 12, 6, 1201-1222.

Aleskerov F.T., Chistyakov V.V., Kalyagin V.A. (2010b). Social Threshold Aggregations. Social Choice and Welfare, 35, 4, 627-646.

Aleskerov F.T., Ersel H., Yolalan R. (2014). Multicriterial Ranking Approach for Evaluating Bank Branch Performance. International Journal of Information Technology and Decision Making, 13, 2, 321-335.

Aleskerov F.T., Yakuba V.I. (2007). A Method for Threshold Aggregation of ThreeGrade Rankings. Doklady Mathematics, 75, 1, 322-324 (in Russian).

Bridges D.S., Mehta G.B. (1995). Representations of Preferences Orderings. Lecture Notes in Economics and Mathematical Systems (LNE, Vol. 422). Berlin: Springer-Verlag.

Chistyakov V.V. (2009). The Enumerating Preference Function in the Multicriterial Problem of the Threshold Aggregation. In: "Functions Theory, its Applications and Related Topics". Works of N. I. Lobachevskii Mathematical Center, 38, 304-306. Kazan: Kazan Mathematical Society (in Russian).

Chistyakov V.V. (2014). On the Superposition of the Borda and Threshold Preference Orders for Three-Graded Rankings. Procedia Computer Science, 31, 1032-1035.

Chistyakov V.V., Kalyagin V.A. (2008). A Model of Noncompensatory Aggregation with an Arbitrary Collection of Grades. Doklady Mathematics, 78, 1, 617-620 (in Russian).

Goncharov A.A., Chistyakov V.V. (2012). Ratings without Compensations and their Application. Control Sciences, 2, 45-52 (in Russian).

d'Aspremont C., Gevers L. (2002). Social Welfare Functionals and Interpersonal Comparability. In: "Handbook of Social Choice and Welfare", 1, 459-541.

Fishburn P.C. (1970). Utility Theory for Decision Making. New York: John Wiley \& Sons. 
Fishburn P.C. (1975). Axioms for Lexicographic Preferences. The Review of Economic Studies, 42, 3, 415-419.

Fishburn P.C. (1999). Preference Structures and their Numerical Representations. Theoretical Computer Science, 217, 359-383.

Graham R.L., Knuth D.E., Patashnik O. (1994). Concrete Mathematics: A Foundation for Computer Science. Reading: Addison-Wesley Publishing.

Hausdorff F. (2005). Set Theory. Third Edition. Providence: Chelsea Publishing, AMS.

Moulin H. (1988). Axioms for Cooperative Decision Making. Cambridge: Cambridge University Press.

Podinovskii V.V. (1975). Multi-Criterion Problems with Uniform Equivalent Criteria. USSR Computational Mathematics and Mathematical Physics, 15, 47-60 (in Russian).

Sen A.K. (1970). Collective Choice and Social Welfare. San Francisco: Holden-Day.

Vilkas E. (1986). An Axiomatic Definition of the Leximin. European Journal of Political Economy, 2, 4, 455-463.

Received 2.07.2018

\section{ЛИТЕРАТУРА}

Алескеров Ф.Т., Якуба В.И. (2007). Метод порогового агрегирования трехградационных ранжировок // Доклады Академии наук. Т. 413. № 2. C. $181-183$.

Гончаров А.А., Чистяков В.В. (2012). Рейтингования без компенсаций и их применение / / Проблемы управления. № 2. С. 45-52.

Подиновский В.В. (1975). Многокритериальные задачи с однородными равноценными критериями / / Журнал вычислительной математики и математической физики. № 2. С. 330-344.

Чистяков В.В. (2009). Функция перечисления в многокритериальной задаче порогового агрегирования. В кн.: “Теория функиий, ее приложения и смежные вопросъ". Труды математического центра им. Н.И. Лобачевского. Т. 38. С. 304-306. Казань: Изд-во «Казанское математическое общество».

Чистяков В.В., Калягин В.А. (2008). Модель некомпенсаторного агрегирования с произвольным набором оценок / / Доклады Академии наук. Т. 421. № 5. C. 607-610.

Aleskerov F., Bouyssou D., Monjardet B. (2007). Utility Maximization, Choice and Preference. Berlin: Springer-Verlag.

Aleskerov F., Chistyakov V.V., Kalyagin V. (2010a). The Threshold Aggregation // Economics Letters. Vol. 107. No. 2. P. 261-262.

Aleskerov F., Yakuba V., Yuzbashev D. (2007). A ‘Threshold Aggregation' of ThreeGraded Rankings // Mathematical Social Sciences. Vol. 53. No. 1. P. 106-110.

Aleskerov F.T., Chistyakov V.V. (2013a). The Threshold Decision Making / / Procedia Computer Science. Vol. 17. P. 1103-1106.

Aleskerov F.T., Chistyakov V.V. (2013b). The Threshold Decision Making Effectuated by the Enumerating Preference Function / / International Journal of Information Technology and Decision Making. Vol. 12. No. 6. P. 1201-1222. 
Aleskerov F.T., Chistyakov V.V., Kalyagin V.A. (2010b). Social Threshold Aggregations / / Social Choice and Welfare. Vol. 35. No. 4. P. 627-646.

Aleskerov F.T., Ersel H., Yolalan R. (2014). Multicriterial Ranking Approach for Evaluating Bank Branch Performance / / International Journal of Information Technology and Decision Making. Vol. 13. No. 2. P. 321-335.

Bridges D.S., Mehta G.B. (1995). Representations of Preferences Orderings. Lecture Notes in Economics and Mathematical Systems (LNE, vol. 422). Berlin: Springer-Verlag.

Chistyakov V.V. (2014). On the Superposition of the Borda and Threshold Preference Orders for Three-Graded Rankings // Procedia Computer Science. Vol. 31. P. 1032-1035.

d'Aspremont C., Gevers L. (2002). Social Welfare Functionals and Interpersonal Comparability. In: "Handbook of Social Choice and Welfare". Vol. 1. P. 459-541.

Fishburn P.C. (1970). Utility Theory for Decision Making. New York: John Wiley \& Sons. [Русск.: Фишберн П. (1978). Теория полезности для принятия решений. Москва: Физматлит.]

Fishburn P.C. (1975). Axioms for Lexicographic Preferences / / The Review of Economic Studies. Vol. 42. No. 3. P. 415-419.

Fishburn P.C. (1999). Preference Structures and their Numerical Representations // Theoretical Computer Science. Vol. 217. P. 359-383.

Graham R.L., Knuth D.E., Patashnik O. (1994). Concrete Mathematics: A Foundation for Computer Science. Reading: Addison-Wesley Publishing.

Hausdorff F. (2005). Set Theory. Providence: Chelsea Publishing, AMS.

Moulin H. (1988). Axioms for Cooperative Decision Making. Cambridge: Cambridge University Press.

Sen A.K. (1970). Collective Choice and Social Welfare. San Francisco: Holden-Day.

Vilkas E. (1986). An Axiomatic Definition of the Leximin // European Journal of Political Economy. Vol. 2. No. 4. P. 455-463.

Поступила в редакиию 2 июля 2018 г.

\section{В.В.Чистяков}

Национальный исследовательский университет Высшая школа экономики, Нижний Новгород

К.О.Чумакова

Национальный исследовательский университет Высшая школа экономики, Нижний Новгород

\section{Восстановление классов безразличия по порядковым номерам при дискретных упорядочениях предпочтения лексимин и лексимакс}

Аннотация. Упорядочение предпочтения лексимин (лексимакс) сравнивает два $n$-мерных вещественных вектора по правилу: вначале координаты этих векторов упорядочиваются по возрастанию (убыванию) и затем получившиеся два вектора сравниваются лексикографически. Хорошо известно, что упорядо- 
чение предпочтения лексимин (лексимакс) не представимо на $\mathbb{R}^{n}$ (какой-либо функцией полезности). В настоящей работе для целых чисел $n \geq 1$ и $m \geq 2$ рассматривается множество $X$ всех $n$-мерных векторов с целыми координатами, принимающими значения между 1 и $m$. Снабдив $X$ упорядочением предпочтения лексимин (лексимакс), индуцированным из $\mathbb{R}^{n}$ и называемым пороговым (двойственным пороговым) правилом, каждому вектору из $X$ (и его классу безразличия) канонически присваивается единственный порядковый номер таким образом, что вектор из $X$ считается более предпочтительным в смысле лексимин (лексимакс), если он лежит в классе безразличия с бо́льшим порядковым номером. Представлен строго обоснованный рекурсивный алгоритм для вычисления кратностей координат вектора из $X$ на основе порядкового номера класса безразличия по отношению к рассматриваемому упорядочению, которому этот вектор принадлежит. Наш алгоритм является новым в двух аспектах: во-первых, он выявляет новые свойства классических биномиальных коэффициентов во взаимодействии с упорядочением предпочтения лексимин (лексимакс) и, во-вторых, он опирается на четыре целочисленных параметра, каждый из которых получается в результате своей индивидуальной циклической процедуры. Совместная работа этих процедур базируется на нашей основной теореме, касающейся некоторых тонких свойств функции перечисления, которая представляет упорядочение предпочтения лексимин (лексимакс) на $X$.

Ключевые слова: слабый порядок, класс безразличия, лексикографическое предпочтение, лексимин, лексимакс, порядковый номер, функиия перечисления. Классификация JEL: C020, C810, D790, E190.

DOI: $10.31737 / 2221-2264-2018-39-3-1$ 